\title{
Newly incident cannabis use in the United States, 2002-2011: A regional and state level benchmark
}

\author{
Jacob P Leinweber ${ }^{1}$, Hui G Cheng ${ }^{1}{ }^{\text {, Catalina Lopez-Quintero }}{ }^{2}$, James C Anthony ${ }^{\text {Corresp. } 1}$ \\ 1 Department of Epidemiology \& Biostatistics, Michigan State University, East Lansing, Michigan, United States \\ 2 Substance Use and HIV Neuropsychology Lab, Center for Children and Families, Miami, Florida, United States \\ Corresponding Author: James C Anthony \\ Email address: janthony@msu.edu
}

Background. Cannabis use and cannabis regulatory policies recently re-surfaced as noteworthy global research and social media topics, including claims that Mexicans have been sending cannabis and other drug supplies through a porous border into the United States. These circumstances prompted us to conduct an epidemiological test of whether the states bordering Mexico had exceptionally large cannabis incidence rates for 2002-2011. The resulting range of cannabis incidence rates disclosed here can serve as 2002-2011 benchmark values against which estimates from later years can be compared.

Methods. The population under study is 12-to-24-year-old non-institutionalized civilian community residents of the US, sampled and assessed with confidential audio computer-assisted self-interviews (ACASI) during National Surveys on Drug Use and Health, 2002-2011 (aggregate $n \sim 420,000$ ) for which public use datasets were available. We estimated state-specific cannabis incidence rates based on independent replication sample surveys across these years, and derived meta-analysis estimates for 10 pre-specified regions, including the Mexico border region.

Results. From meta-analysis, the estimated annual incidence rate for cannabis use in the Mexico Border Region is $5 \%$ ( $95 \%$ confidence interval, $\mathrm{Cl}=4 \%, 7 \%$ ), which is not an exceptional value relative to the overall US estimate of $6 \%(95 \% \mathrm{Cl}=5 \%, 6 \%)$. Geographically quite distant from Mexico and from states of the western US with liberalized cannabis policies, the North Atlantic Region population has the numerically largest incidence estimate at $7 \%(95 \% \mathrm{Cl}=6 \%, 8 \%)$, while the Gulf of Mexico Border Region population has the lowest incidence rate at $5 \%(95 \% \mathrm{Cl}=4 \%, 6 \%)$. Within the set of state-specific estimates, Vermont's and Utah's populations have the largest and smallest incidence rates, respectively (VT: 9\%; 95\% Cl = 8\%, 10\%; UT: 3\%; 95\% Cl = 3\%, 4\%).

Discussion. Based on this study's estimates, among 12-to-24-year-old US community residents, an estimated 6 percent start to use cannabis each year (roughly 1 in 16). Relatively minor variation in region-wise and state-level estimates is seen, although Vermont and Utah might be exceptional. As of 2011, proximity to Mexico, to Canada, and to the western states with liberalized policies apparently has induced little variation in cannabis incidence rates. Our primary intent was to create a set of benchmark estimates for state-specific and region-specific population incidence rates for cannabis use, using metaanalysis based on independent US survey replications. Public health officials and policy analysts now can use these benchmark estimates from 2002-2011 for planning, and in comparisons with newer estimates. 
Please do not cite or quote before publication

Newly incident cannabis use in the United States, 2002-2011:

\section{A regional and state level benchmark}

Jacob P Leinweber, Hui G Cheng, Catalina Lopez-Quintero, James C Anthony

Department of Epidemiology \& Biostatistics

Michigan State University, East Lansing, Michigan, USA

*Address correspondence to

James C Anthony, Ph.D.,

Professor, Department of Epidemiology and Biostatistics,

Michigan State University, East Lansing, Michigan, USA

E-mail: janthony@msu.edu 


\section{Abstract}

1 Background. Cannabis use and cannabis regulatory policies recently re-surfaced as noteworthy

2 global research and social media topics, including claims that Mexicans have been sending

3 cannabis and other drug supplies through a porous border into the United States. These

4 circumstances prompted us to conduct an epidemiological test of whether the states bordering

5 Mexico had exceptionally large cannabis incidence rates for 2002-2011. The resulting range of

6 cannabis incidence rates disclosed here can serve as 2002-2011 benchmark values against which

7 estimates from later years can be compared.

8 Methods. The population under study is 12-to-24-year-old non-institutionalized civilian

9 community residents of the US, sampled and assessed with confidential audio computer-assisted self-interviews (ACASI) during National Surveys on Drug Use and Health, 2002-2011

11 (aggregate $\mathrm{n} \sim 420,000$ ) for which public use datasets were available. We estimated state-specific cannabis incidence rates based on independent replication sample surveys across these years, and derived meta-analysis estimates for 10 pre-specified regions, including the Mexico border region.

Results. From meta-analysis, the estimated annual incidence rate for cannabis use in the Mexico Border Region is 5\% (95\% confidence interval, $\mathrm{CI}=4 \%, 7 \%)$, which is not an exceptional value relative to the overall US estimate of $6 \%(95 \% \mathrm{CI}=5 \%, 6 \%)$. Geographically quite distant from Mexico and from states of the western US with liberalized cannabis policies, the North Atlantic Region population has the numerically largest incidence estimate at $7 \%(95 \% \mathrm{CI}=6 \%, 8 \%)$, while the Gulf of Mexico Border Region population has the lowest incidence rate at 5\% (95\% CI

$21=4 \%, 6 \%$ ). Within the set of state-specific estimates, Vermont's and Utah's populations have the 22 largest and smallest incidence rates, respectively (VT: 9\%; 95\% CI =8\%, 10\%; UT: 3\%; 95\% CI 
$23=3 \%, 4 \%)$.

24 Discussion. Based on this study's estimates, among 12-to-24-year-old US community residents,

25 an estimated 6 percent start to use cannabis each year (roughly 1 in 16). Relatively minor

26 variation in region-wise and state-level estimates is seen, although Vermont and Utah might be

27 exceptional. As of 2011, proximity to Mexico, to Canada, and to the western states with

28 liberalized policies apparently has induced little variation in cannabis incidence rates. Our

29 primary intent was to create a set of benchmark estimates for state-specific and region-specific

30 population incidence rates for cannabis use, using meta-analysis based on independent US

31 survey replications. Public health officials and policy analysts now can use these benchmark

32 estimates from 2002-2011 for planning, and in comparisons with newer estimates. 


\section{Introduction}

33 Drug supply and availability occupy central positions among environmental conditions and

34 processes that account for risk of becoming a drug user, with a potentially cascading influence on transitions from newly incident drug use toward associated problems, such as drug dependence syndromes or addiction states (Volkow \& Li, 2005). In research on cannabis (marijuana, marihuana), a recent illustration can be seen from evidence on twins and college students born within the United States (US), which supports this view of cannabis availability and 'exposure opportunities' as major environmental influences on becoming a cannabis user, and on occurrence of cannabis problems, once cannabis use starts (Gillespie, Neale \& Kendler, 2009; Pinchevsky et al., 2012).

These ideas about 'drug exposure opportunities' and associated environmental variations emerged from Wade Hampton Frost's early conceptualization of epidemiology as a population science, including his specification of an interacting 'agent-host-environment' triad that now guides public health research generally (Frost, 1928, reprinted 1976). In Frost's triad model, the 'agent' functions as a necessary but not sufficient cause, and motivates epidemiological attention to 'hosts' who live close by environmental 'reservoirs' that support agent viability and propagation - that is, the origin or sources of 'supply' from which agents are conveyed until they make effective contact with susceptible hosts. Hosts living in geographical areas that are distant from an agent's reservoir, with limited or no 'exposure opportunity' for that agent, should have

51 lower incidence rates. These hosts should be less likely to become newly infected or newly

52 incident cases of the disease, whereas those living close by the agent's reservoir might well have 53 more exposure opportunities, as well as associated greater risk for becoming infected. Wagner 54 and Anthony (2002) extended Frost's concepts, by analogy, to cannabis and other drug use. 
55 For several reasons, definitive evidence on the 'cannabis reservoir' and associated 'place by

56 place' geographic variations in cannabis availability within the US is in short supply. Drug law

57 enforcement agencies do not yet take a systematic approach to 'controlled buys,' as might be

58 used to discriminate area-specific prices charged at retail versus wholesale levels (Manski,

59 Pepper \& Petrie, 2001). Furthermore, in contrast to epidemiological research on tobacco

60 cigarettes, studies of internationally regulated drugs of illegal origin do not include a common

61 metric of 'one cigarette' and 'one pack' that can be used to construct pack-years of personal

62 exposure; state and substate jurisdictional tax receipts are not yet useful. For reasons of this type,

63 many drug researchers have raised serious questions about the evidence base required to evaluate

64 the impact of federal or state drug policies regulating drug supply and availability (Manski,

65 Pepper \& Petrie, 2001). In a more recent critique prepared for the US federal government and

66 focused on cannabis specifically, scientists working for the RAND Corporation used the phrase

67 'lacks credibility' when describing the federal approach now used to estimate the amount of

68 cannabis available in US markets (United States, 2012c).

69 Notwithstanding critiques along these lines, against a background of increasing domestic crop

70 yield within US borders, it is possible to draw a fairly firm conclusion that Mexico is the country

71 that has been supplying most cannabis to the US market, by volume. According to a series of

72 World Drug Reports contributed by the United Nations Office on Drugs and Crime, in addition

73 to cannabis trafficking flowing primarily from Mexico into the United States, both Mexico and

74 the US have been the top two countries in cannabis herb seizures since the turn of the $21^{\text {st }}$

75 century. These two countries alone have made up at least one third of all seizures worldwide

76 (United Nations Office of Drug and Crime, 2016). In contrast, to the extent that available law

77 enforcement data can be trusted for rough comparative purposes, the Canadian supply has been 
78

characterized as 'small' (United States, 2012c).

Figure 1 helps substantiate what now is known about the 'cannabis reservoir' relative to the geography of the United States. It is adapted from an illustration prepared by the US Library of

Congress Federal Research Division and National Drug Intelligence Center. It confirms

Mexico's position as a major source country in its depiction of both land and sea cross-border trafficking routes for cannabis. Nevertheless, it should be noted that the map leaves out what is presumed to be much more limited trafficking via governmental postal systems and international flights (United States Library of Congress, 2003).

Coupled with prevailing theories about importance of cannabis availability, this type of map leaves an impression that cannabis availability, and possibly the incidence rates for becoming a newly incident cannabis user, might be greater for populations living along the US border with Mexico, relative to other regions of the US. Accordingly, we set out to investigate this speculative proposition, with a deliberately naïve advance expectation that the incidence rates for cannabis use might be greater in the states along the US-Mexico border (as depicted West to East in Figure 1: California, Arizona, New Mexico, and Texas).

Of course, in this work, we had to allow for the possibility that proximity to the cannabis croplands of Mexico might not be the only governing influence. This logic prompted us to set aside California from our originally pre-specified regional grouping of border states, given that the California population has had a tradition of relatively liberalized views about cannabis and other drug use. In addition, California shares a Pacific Ocean coastline with Mexico. Northern California has its own extensive cannabis croplands. Finally, there is an extensive set of roads linking northern California with Oregon across these two western US states with well- 

documented progressive politics.

101 In the background, we also had to take into account the possibility that cross-border smugglers 102 might make a better profit by driving their just-smuggled cannabis to a non-border state, in 103 which case street-level retail profit margins for cannabis might lead to greater incidence in non104 border states distant from Mexico, such as Nevada, Colorado, Utah, and Oklahoma (Manski, 105 Pepper \& Petrie, 2001). For reasons of this type, our presentation of state-level estimates in this 106 paper makes it possible for readers to evaluate and re-configure our regional specifications as a 107 check on what we drew up before any analyses. In addition, the authors can provide CSV and 108 Stata .dta data files with state-level estimates, as well as their standard errors, so that interested readers can study the state-level estimates and can re-configure the regional specifications as 110 they see fit.

111 In this line of research on cannabis incidence rate estimates, several prior contributions deserve 112 attention, but have not addressed US region-wise or state-specific cannabis incidence rates 113 directly. To illustrate, Rhodes and colleagues (2003) provided a fairly comprehensive overview 114 of major determinants of cannabis smoking, over and above the issue of border proximity and 115 area variations in availability, price, and regulations (Rhodes et al., 2003). In this regard, other macro-environmental factors that vary state by state, such as higher levels of poverty, lower 117 education attainment, more rapid population growth, and marked social inequalities might contribute to variations in incidence rates for cannabis use onset among young people in various 119 regions and states of the US (Bruhn, 2014; Ganster \& Lorey, 2008). In addition, there are state120 specific estimates for the prevalence of cannabis smoking (Hughes, Lipari \& Williams, 2013; 
121 United States, 2012a). The published prevalence estimates are from surveys of 12-to-17-year-

122 olds and 18-to-25-year olds in 2010-14, and convey estimated state-specific proportions for

123 individuals with any cannabis smoking at any time during the year prior to the date of survey

124 assessment. The observed patterns in these state-specific prevalence estimates have suggested

125 that the US states bordering Mexico might be remarkable for excess prevalence, with the

126 possible exception of Texas, and have helped substantiate predictions made by others (e.g.,

127 Harrison \& Kennedy, 1994).

128 Limits on the definitiveness of these published prevalence estimates can be noted by observing

129 that 'prevalence' is a rather complex multi-component parameter governed by (a) forces that

130 influence 'becoming a newly incident user' as well as (b) forces that influence the 'duration' or

131 persistence of use, once drug use has started. This complexity of prevalence as a summary

132 population statistic in health research is a basic epidemiological principle noted more than 50

133 years ago by Lapouse, among others. In her work, Lapouse drew attention to the limited utility of

134 prevalence estimates when the aim is to study influences on health, for which knowledge of

135 newly incident cases is required. She recommended restricting the use of prevalence estimates to

136 the planning of health services because prevalence conveys an impression of the total caseload

137 facing a community without discriminating whether the cases are chronic or long-sustained

138 versus newly incident (Lapouse, 1967). In research on drug use, it is correct to say that

139 prevalence of drug use serves as an epidemiological indicator of the theoretical 'market demand'

140 for a drug because the market demand segments include both newly incident users observed in

141 any given year, as well as long-time persistent users with drug onsets in prior years (Cheng,

142 Cantave \& Anthony, 2016). In this paper, we make no direct comparison of cannabis incidence

143 rates and cannabis prevalence proportions. Nevertheless, for readers who might be interested in 
144 the comparison of state-level prevalence and incidence estimates, color gradient 'heat maps' of

145 state-specific cannabis prevalence estimates for the US can be found elsewhere (United States, 146 2012a).

147 In this research report, we focus strictly upon the incidence rate for becoming a newly incident

148 cannabis user (i.e., with focus on individuals who started using cannabis for the first time during

149 the year prior to the date of survey assessment). This focus on newly incident users reflects our

150 attempt to ask a more specific research question about regional patterns for observed risk of first

151 time use of cannabis in the US-Mexico border state populations relative to incidence rate

152 estimates for other US regions, and to create state-level benchmark values for use in public

153 health policy and program planning. In an online report based on survey estimates through 2014,

154 Lipari and colleagues (Lipari, Kroutil \& Pemberton, 2015) recently provided estimates of the

155 number of new cannabis initiates (in millions) and for several age subgroups (e.g., 12-to-17-year-

156 olds), but did not convert these numbers to incidence rates and did not supply state-specific

157 incidence rate estimates, which we present for the first time in the peer-reviewed journal

158 literature.

\section{Materials and Methods}

\subsection{Study Population and Samples}

159 For this research, we turned to nationally representative survey samples drawn and assessed each

160 year from 2002 through 2011 for the National Surveys on Drug Use and Health (NSDUH). In

161 these surveys, each state's sample in a given year is a statistically independent replication

162 sample, with state-specific numbers of sample participants guided by the size of the state 
163 population. Each year's NSDUH study population is specified to consist of non-institutionalized

164 civilian Americans aged 12+ years residing in the 50 states or the US District of Columbia. Each

165 year, multi-stage area probability sampling approaches encompass residents of non-institutional

166 group quarters (e.g., homeless shelters, college dormitories) as well as residents of household

167 dwelling units. State Sampling Regions (SSRs) are created in each state based on census data.

168 From within SSRs, probability sampling is used to select the dwelling units (DU) and the

169 rostered inhabitants of each DU, from which a probability sample of designated survey

170 respondents is drawn. The NSDUH field staff are responsible for visiting and securing a roster

171 for each designated DU, and then for introducing and securing informed consent or assent from

172 each designated respondent. The resulting NSDUH samples from 2002 through 2011 have

173 included more than 60,000 designated respondents recruited after informed consent protocols

174 approved by cognizant committees for protection of human subjects ( $\mathrm{n}$ 420,000 12-to-24-year-

175 olds). Participation levels have been between $70 \%$ and $80 \%$ during these years (see

176 supplementary material for a more detailed description). Additional details about NSDUH are

177 provided in online monographs (United States, 2012b; United States, 2015) and many published

178 articles (e.g., Cheng, Cantave \& Anthony, 2016).

179 This research project's estimates are based upon the public use datasets created from the 180 NSDUH study operations, which have been termed the Restricted-Data Analysis System (R-

181 DAS), as described in detail in a prior study of state-level estimates for prescription pain-killers

182 (Vsevolozhskaya \& Anthony, 2015). For this study’s cannabis estimates, we turned to R-DAS

183 10-year datasets for online analyses of the NSDUH 2002-2011 data, with analysis weights

184 crafted for that 10 year interval. Because age-specific incidence of cannabis use drops to 
185 extremely low levels at age 24 years, we focused estimation on the subgroup of 12-to-24-year-

186 old young people, yielding an aggregate 10 year unweighted sample size of approximately

187420,000 individuals, with no need for age-standardization adjustments given relatively balanced

188 state-by-state age distributions in this age range (United States, 2012b; United States, 2015). [In

189 addition, given state-specific imbalanced distributions at older ages (e.g., population proportion

190 age 50 years and older in the US-Mexico border states versus US-Canada border), the entire age

191 range of NSDUH participants was not considered.]

\subsection{Assessment}

192 NSDUH employs audio computer-assisted self-interviews (ACASI) to promote accuracy and completeness of self-reports about drug use and related behaviors. Newly incident cannabis users are identified via a specific module that asks about month and year of first cannabis use, with results recorded in an R-DAS variable called RECMJ_B. Cross-classification of RECMJ_B with the R-DAS variable ELGMJ_B makes it possible to identify individuals who were 'at risk' of starting to use cannabis for the first time during the 12 months prior to assessment, with differentiation of those who did or did not start using during that interval.

The covariates of central interest are US geographic regions crafted by our research team. Prior to analysis, we sorted each state into ten regions pre-specified according to our judgments about potential availability and access to cannabis via neighboring land or water borders. After creating

202 initial regions around the borders of the United States, all remaining states were grouped together into one interior region. Prior to analyses, we re-sorted some states and removed them from

204 border regions as described in the next paragraph. 
205 For our pre-analysis specification of US regions, Alaska and Hawaii were set as 'regions' in and 206 of themselves. All US-Mexico border states (except for California) were placed in one region.

207 California was aggregated with Oregon and Washington State based on the 'Pacific Ocean

208 border' considerations described in our introduction. However, once we considered Washington

209 State's position on the Pacific Ocean and also along the US-Canada border, we placed it into its

210 own single-state region, and placed all other states along the Canadian border in a US-Canada

211 border region (e.g., from Maine to Idaho, including New York State). All other states along the

212 Atlantic Ocean were split into North and South regions based upon where the Chesapeake Bay

213 estuary flows into the Atlantic Ocean. The states along the Gulf of Mexico were aggregated,

214 including Florida (despite its Atlantic shoreline). All remaining states were grouped in a single

215 region as 'interior' or 'non-border' states with neither national borders nor ocean shorelines

216 protected by the US Coast Guard, Customs, or Justice Department agents who now enforce US

217 cannabis regulations as part of Homeland Security protections.

218 As noted, one goal of these pre-assignments was to address the fact that some states certainly

219 might qualify for membership in multiple regions defined by border considerations. We have a

220 statistical rationale for beginning by placement of each state into only one and only one

221 aggregated region, or leaving it by itself as in the case of Washington State. A resulting statistical

222 advantage is reduced complexity when making incidence rate comparisons across each

223 independently specified region. In this fashion, covariances due to duplicate entries are

224 eliminated.

225 We ended up with an initial pre-specification of 10 regions (i.e., specified before estimation), as

226 follows: (1) Alaska; (2) Hawaii; (3) Mexico Border Region (Texas, New Mexico, and

227 Arizona; minus California); (4) Canada Border Region (Idaho, Montana, North Dakota, 
228 Minnesota, Wisconsin, Illinois, Indiana, Michigan, Ohio, Pennsylvania, New York, Vermont,

229 New Hampshire, and Maine; minus Washington State); (5) North Atlantic Border Region

230 (Massachusetts, Connecticut, Rhode Island, New Jersey, Delaware, and Maryland); (6) South

231 Atlantic Border Region (Virginia, North Carolina, South Carolina, and Georgia); (7) Gulf of

232 Mexico Border Region (Florida, Alabama, Mississippi, Louisiana); (8) Pacific Border Region

233 (California and Oregon); (9) Washington State; (10) Non-Border States (Nevada, Colorado,

234 Utah, Wyoming, Nebraska, South Dakota, Oklahoma, Missouri, Kansas, Iowa, Tennessee,

235 Arkansas, Kentucky, West Virginia, and District of Columbia). A small number of controversial

236 assignments have been addressed via post-estimation re-assignments. For example, in a post-

237 estimation analysis we have combined California in a re-assignment with the other US-Mexico

238 border states.

\subsection{Analysis}

239

240

241

242

243

244

245

246

247

This study's cannabis incidence rate for 12-to-24-year-olds was estimated, state-by-state, as the weighted number of newly incident users divided by the weighted number of 'at risk' individuals (i.e., never users plus newly incident users), based on R-DAS analysis weights for the 10 year interval. We then used meta-analysis to group the states by region and to produce a regionspecific summary estimate, with a random effects estimator when heterogeneity chi-square test statistics disclosed a heterogeneity I-squared statistic $>0.50$ with $\mathrm{p}<0.05$ (DerSimonian \& Laird, 1986; Higgins et al., 2003). Given that state-specific sample sizes are drawn proportional to state population size, all standard errors for state-specific estimates are 'information' weighted. That is, states with larger populations have larger samples. This fact is reflected in the 
248 region-wise meta-analysis summary estimates. Finally, a meta-analysis summary estimate for the

249 US as a whole was derived on the basis of the region-specific estimates.

250 The R-DAS analysis weights for the 10 year interval are used to take into account both sample

251 selection probabilities and post-stratification adjustment factors based upon US Census

252 subpopulation counts. Standard errors and 95\% confidence intervals (CI) are from complex

253 survey delta methods. Statistical significance was examined with two indicators that are useful in

254 the large sample context. First, when comparing two independent (mutually exclusive) state-

255 specific or region-specific estimates, an overlap of the two 95\% CI usually indicates the null,

256 whereas non-overlap typically suggests a non-null difference with analogous non-null-p-value.

257 Next, we performed formal z-test comparisons, following instructions laid out by the NSDUH

258 method group (United States, 2016), to assess whether there are any non-null differences

259 between the Mexican border region and other regions that might have been missed using the

$26095 \%$ CIs method. Second, the meta-analysis heterogeneity test statistic at $\mathrm{p}<0.05$ signifies

261 substantial within-region variation (i.e., among the several states in the region). All meta-

262 analyses have been completed using the statistical software Stata Version 13.1 (StataCorp,

263 College Station, Texas, USA, 2014).

\section{Results}

264 Figure 2 shows region-specific estimated incidence rates for becoming a newly incident user of

265 cannabis among 12-to-24-year-old community residents, and a pattern that falsifies our initial

266 expectation. The US-Mexico border region, specified to include Texas, New Mexico, and

267 Arizona, has mid-range cannabis incidence at 5\% per year $(95 \% \mathrm{CI}=4 \%, 7 \%)$, relative to the 
268 US summary estimate of $6 \%$ per year. In a post-estimation re-aggregation, we joined California

269 with these three states (given its common Mexico border), and derived a new incidence estimate

270 of $6 \%(95 \% \mathrm{CI}=5 \%, 7 \%)$, still mid-range (data shown in supplementary raw data file). Our

271 conclusion remains the same according to results from the forma $\mathrm{z}$-test comparison

272 (Supplementary Table 3). That is, no differences are found between the US-Mexico border

273 region and each of the other regions.

274 Figure 2 also shows that, numerically, the largest cannabis incidence rate is seen in the North

275 Atlantic region at $7 \%$ per year $(95 \% \mathrm{CI}=6 \%, 8 \%)$ and in the Pacific Border region (California

276 plus Oregon) at 7\% $(95 \% \mathrm{CI}=5 \%, 8 \%)$ In a post-estimation re-specification, we joined

277 Washington State within the Pacific Border aggregate. Relative to the observed range, the

278

279

280

281

282

283

284

285

286

287

288

289

290 resulting new incidence estimate of $6 \%(95 \% \mathrm{CI}=6 \%, 7 \%)$ remains at the higher end, but it is numerically lower than the $7 \%$ estimate for the California-Oregon aggregate (data shown in supplementary raw data file).

Readers interested in the state-specific estimates or in re-aggregating the states with different regional specifications may be interested in Figures 3 and 4, and will find the map's statespecific estimates in our online supplemental materials. Figure 3 is a heat map, created with the free online software OpenHeatMap, for which each state's color depends on its annual cannabis use incidence estimate, such that the northeastern states are depicted as much darker than the southern states (Warden, OpenHeatMap, 2010). Figure 4 is a cartogram or 'blob' map for which each state's relative size is depicted as a function of its annual cannabis use incidence estimate, such that the northeastern states are depicted as much larger than in an area-size depiction. The cartogram was constructed with the mapping software ScapeToad Version 1.2 (Lévy et al., Chôros Laboratory, Lausanne, Switzerland, 2008). The maps and the supplemental state-specific 
291 estimates disclose that the 12-to-24-year-old population in Vermont has the largest cannabis

292 incidence rate at $9 \%$ per year $(95 \% \mathrm{CI}=8 \%, 10 \%)$. The smallest incidence is seen in Utah at 3\%

293 per year $(95 \% \mathrm{CI}=3 \%, 4 \%)$, shown in Figure 4 as a small L-shaped state just above the Mexico

294 border states. As described in Section 2, Utah was pre-classified as one of our non-border states;

295 its population has some distinctive characteristics that might play a role in this observed lower

296 cannabis incidence estimate, over and above its distance from US borders and coastlines (e.g.,

297 see Vsevolozhskaya \& Anthony, 2015).

\section{Discussion}

298

299

300

301

302

303

304

305

306

307

308

309

310

The most surprising discovery in this research might be the relative homogeneity of the regionspecific cannabis incidence estimates within the US. For the most part, all region-specific estimates have overlapping confidence intervals. In consequence, we falsified our expectation that living in relative proximity to the US-Mexico border, nearby the cannabis croplands of Mexico, might produce a larger cannabis incidence rate. The population of the US-Mexico border region has a mid-range cannabis incidence estimate, not appreciably different from those of the other region-specific populations studied here. No clear epicenters or geographical gradients can be seen in the region-wise and state-specific estimates.

All prior epidemiological information about cannabis use in the populations of the US-Mexico border states is based upon regional estimates for prevalence of recently active cannabis use. At present, these states do not appear to have exceptional cannabis incidence rates. If other assertions about elevated cannabis prevalence in this region are correct (e.g., Harrison \& Kennedy, 1994; United States, 2012b), but cannabis incidence is not elevated, then the 
311 implication is greater persistence or duration of cannabis use once it gets started (Lapouse, 1967;

312 Cheng, Cantave \& Anthony, 2016).

313 Before any additional discussion of these results, several of the more important study limitations

314 merit attention. The focus on starting to use cannabis in the 12 months prior to assessment

315 constrains the influence of state in- and out-migration on the study estimates, but unrestricted

316 NSDUH datasets included no study variable on the duration of residence in a specific state. For

317 this reason, a small minority of the newly incident users might have started to use in one state,

318 followed by migration into a different state of residence at that time of NSDUH sampling.

319 Some readers might regard our focus on cannabis incidence rates (becoming a cannabis user for

320 the first time) as a limitation. Here, we return to the issues mentioned in our Abstract and

321 Introduction. Namely, in epidemiological analyses of any condition that involves the agent-host-

322 environment triad, the prevalence proportion mixes up two mechanisms: (1) the mechanisms

323 involved with making effective contact with the agent in the first place, and thereby becoming

324 affected by the agent-attributable condition (in this instance, responding to the chance to try

325 cannabis with actual first-time cannabis use), and (2) the separable mechanisms that determine

326 persistence or duration of the condition after it has started (in this instance, duration of cannabis

327 use). We have a project underway in order to evaluate whether living in the Mexico border

328 region might influence the duration or persistence of cannabis use, once it has started, but there

329 are some complexities that limit inferences in that context. One complexity is that we must keep

330 track of how recently the study participant might have moved from a non-border state into a

331 border state, and whether the prevalence of cannabis use might be greater in the border states as a

332 result of seriously involved cannabis users migrating into border states so that they can more 
333 readily acquire a supply of cannabis. This situation became more complex after 2011, with an

334 increasing number of non-border states creating a more liberal cannabis regulatory environment

335 (e.g., in Colorado, Oregon, and Washington State) such that migration of long-duration cannabis

336 users to those states might drive up prevalence in the non-border states with liberalized policies,

337 with possibly no effect in incidence rates. We have asked the NSDUH authorities for access to

338 survey information on pre- and post-cannabis migration of the study participants, but our access

339 to those restricted data has not yet been approved.

340 Another study population issue involves the sampling frame's deliberate exclusion of each

341 state's institutionalized individuals and its military residents. However, this study feature should

342 not have a major influence on estimation of state-specific cannabis incidence rates. Validity and

343 reliability of the survey estimates based upon recalled experience of first cannabis use might vary

344 by state or region (e.g., if influenced by local or state policies governing possession and use, or

345 by law enforcement practices). Nonetheless, we do not consider our findings to be severely

346 biased by this possibility because (a) the recall period is tightly constrained to the most recent 12

347 months of each participant's lifetime, and (b) the use of ACASI enhances participants'

348 willingness to self-disclose use of internationally regulated drugs (United States, 2012b). Indeed,

349 the focus on a readily recalled life event ( $1^{\text {st }}$ cannabis use) within a 12 month time interval of a

350 12-to-24-year-old participant might represent a major strength of this study as compared to

351 alternative approaches.

352 Counterbalancing strengths include the study's large nationally representative survey samples

353 with acceptable (although not ideal) participation levels. Whereas cannabis incidence estimates 
354 from prospective or longitudinal studies might be considered a gold standard, we note that this 355 study's incidence estimates are free of follow-up attrition biases and involve no measurement

356 reactivity of the type that is faced when an individual is assessed repeatedly in a longitudinal

357 design such that the answers in an earlier assessment can influence answers in a later assessment 358 (Anthony, 2010). Finally, when compared to the information value of previous state-specific 359 estimates for cannabis prevalence, this study's use of the incidence parameter constrains 360 potentially confounding influences of in-migration and out-migration after onsets have occurred 361 (Lapouse, 1967).

In future research that builds from findings such as these, the role of other local- and region-level factors (e.g., law enforcement, price, 'medical marijuana' policies, etc.) might be explored in order to find the most relevant meso-level correlates with cannabis incidence in young people. For example, notwithstanding concerns expressed by others (e.g., Manski, Pepper \& Petrie, 2001), there is some research on state- and regional-level variations in price of cannabis

367 products, which could be integrated with incidence analyses (Ruggeri, 2013). In addition, both Mexico and Canada have joined the $21^{\text {st }}$ century trend toward relaxation of penalties for cannabis possession and use (e.g., Freckelton, 2015). Influences on cannabis incidence in the Mexico and Canada border states may extend beyond relative availability and trade across national borders

371 into the domains of perceptions about normative behavior and whether it is very risky to start

372 using drugs; these perceptions have been found to cluster within neighborhoods of US

373 communities, which is evidence of social sharing that might extend across national and state 374 borders (Petronis \& Anthony, 2000). Recent estimates suggest that the passage of medical 375 marijuana law induced null to negative change in the prevalence of cannabis use (Hasin et al., 
376 2015); nonetheless, it is possible that a decades-long research process is required to disclose

377 policy impact of this type on prevalence, given that parameter's multi-component complexity as

378 described in our introduction and by Lapouse (1957). Differences in incidence estimates of

379 cannabis use should provide more immediate and possibly more robust evidence for the potential

380 influence of change in cannabis policies of this type. In summary, even though available maps of

381 cannabis flow now draw attention to Mexico's croplands as major sources of supply and

382 availability of this drug within the US (e.g., Figure 1), the region-specific cannabis incidence

383 estimates of this study falsify our expectation that 12-to-24-year-olds living in Mexico border

384 states might have been more likely to become newly incident cannabis users during the interval

385 from 2002 through 2011. The largest cannabis incidence rate estimates in the United States are

386 not seen in the US-Mexico border region. Rather, it is in Vermont and in the North Atlantic

387 Region that we see the largest estimates.

388 An increasing number of US jurisdictions are removing penalties for 'recreational use' of

389 cannabis. This study's investigation of geographical variations in cannabis incidence rates create

390 'benchmark values' and should provide a useful foundation for a future extension of this line of

391 epidemiological research on 'place' as a potential determinant of starting to use cannabis

392 products. In a recent literature review, Anthony, Lopez-Quintero \& Alshaarawy noted a lack of

393 survey-based incidence estimates for cannabis use around the globe (2017). This study

394 exemplifies such estimation of incidence rates using publicly available national data.

395 State-level public health planners and policy makers might have a more immediate use of this

396 study's estimates, coupled with estimates that roughly $2 \%$-to- $4 \%$ of young people in the US

397 develop a cannabis dependence syndrome within 12-24 months after first cannabis use (Chen,

398 O'Brien \& Anthony, 2005). Taking Utah as an example, that state now has an estimated 
399 population size of close to 500,000 12-to-24-year-olds who have never tried cannabis (data

400 shown in supplementary raw data file). Applying the estimated Utah cannabis annual incidence

401 rate of $3 \%$ per year and the published cannabis dependence case transition probability of just $2 \%$,

402 one might project as many as 15,000 newly incident cannabis users in that state population each

403 year, and a total of 300 newly incident cannabis dependence cases possibly needing intervention

404 services each year. (If the $4 \%$ transition probability holds, then this caseload count of newly

405 incident cannabis dependence cases grows from a forecast of 300 to 600.) If these forecasts are

406 correct, even when the epidemiology of cannabis use and dependence in Utah is in 'steady state,'

407 with no change from these parameter estimates, the Utah public health officials face a substantial

408 number of newly incident cannabis dependence cases in their population of young people each

409 year. Some of these newly incident cannabis users will require outreach and early intervention

410 services of the type that can be used to reduce person-to-person spread of drug use soon after

411 onset of use in an index user (Hughes, Lipari \& Williams, 2013). Applied in this fashion, state-

412 level incidence and case transition probability estimates from epidemiological studies become

413 more than 'weather report' statistics or 'yesterday's news' and can add valuable guidance for

414 practical public health planning activities, as well as for evaluation of the impact of future

415 cannabis policy changes.

\section{Acknowledgments}

416 We are grateful to Zachary Sadler and Adnan Moustapha Barazi for their contributions to this

417 project. 


\section{References}

418

419

420

421

422

423

424

425

426

427

428

429

430

431

432

433

434

435

436

437

438

439

440

441

442

443

444

445

446

447

448

449

450

451

452

453

454

455

456

457

458

459

460

461

Anthony JC. 2010. Novel phenotype issues raised in cross-national epidemiological research on drug dependence. Ann N Y Acad Sci 1187:353-369. 10.1111/j.1749-6632.2009.05419.x.

Anthony JC, Lopez-Quintero C, and Alshaarawy. 2017. Cannabis Epidemiology: A Selective Review. Curr Pharm Des 22(42):6340-6352.

Bruhn JG. 2014. The Border Region: Its Culture and Health Disparities. In Culture and Health Disparities. Cham, Switzerland: Springer International Publishing.

Chen CY, O'Brien MS, and Anthony JC. 2005. Who becomes cannabis dependent soon after onset of use? Epidemiological evidence from the United States: 2000-2001. Drug Alcohol Depend 79(1):11-22.

Cheng HG, Cantave MD, and Anthony JC. 2016. Alcohol experiences viewed mutoscopically: I. Newly incident drinking of 12-25 year olds in the United States, 2002-2013. Journal of Studies on Alcohol and Drugs 77:405-412.

DerSimonian R, and Laird N. 1986. Meta-analysis in clinical trials. Control Clin Trials 7:177188.

Freckelton I. 2015. Medicinal Cannabis Law Reform: Lessons from Canadian Litigation. J Law Med 22:719-738.

Frost WH. 1976. Some conceptions of epidemics in general. Am J Epi 103(2):141-151. (Originally delivered as a Harvard University 'Cutter' lecture in 1928).

Ganster P and Lorey DE. 2008. The U.S.-Mexico border in the 21st century (2nd ed.). Lanham, MD: Rowman \& Littlefield Pub.

Gillespie NA, Neale MC, and Kendler KS. 2009. Pathways to cannabis abuse: a multi-stage model from cannabis availability, cannabis initiation and progression to abuse. Addiction 104:430-438. 10.1111/j.1360-0443.2008.02456.x

Harrison LD and Kennedy NL. 1994. Drug use in the United States-Mexico border area: Is there an epidemic waiting to happen? Hispanic Journal of Behavioral Sciences 16(3):281-295.

Hasin DS, Wall M, Keyes KM, Cerda M, Schulenberg J, O'Malley PM, Galea S, Pacula R, and Feng T. 2015. Medical marijuana laws and adolescent marijuana use in the USA from 1991 to 2014: results from annual, repeated cross-sectional surveys. Lancet Psychiatry 2:601-608. 10.1016/S2215-0366(15)00217-5

Higgins JP, Thompson SG, Deeks JJ, and Altman DG. 2003. Measuring inconsistency in metaanalyses. BMJ 327:557-560. 10.1136/bmj.327.7414.557

Hughes A, Lipari RN, and Williams M. 2013. State Estimates of Adolescent Marijuana Use and Perceptions of Risk of Harm From Marijuana Use: 2013 and 2014. The CBHSQ Report. Rockville, MD.

Lapouse R. 1967. Problems in studying the prevalence of psychiatric disorder. Am J Public Health Nations Health 57:947-954.

Lipari R, Kroutil LA, and Pemberton MR. 2015. Risk and protective factors and initiation of substance use: Results from the 2014 National Survey on Drug Use and Health. Online report: https://www.samhsa.gov/data/sites/default/files/NSDUH-DR-FRR42014rev/NSDUH-DR-FRR4-2014.pdf, last accessed 17 March 2017.

Manski CF, Pepper JV, and Petrie CV. 2001. Informing America's Policy on Illegal Drugs. What We Don't Know Keeps Hurting Us. Washington DC, USA: NATIONAL ACADEMY PRESS.

Petronis KR, and Anthony JC. 2000. Perceived risk of cocaine use and experience with cocaine: 
462

463

464

465

466

467

468

469

470

471

472

473

474

475

476

477

478

479

480

481

482

483

484

485

486

487

488

489

490

491

492

493

494

495

496

497

498

499

500

501

502

503

504

505

do they cluster within US neighborhoods and cities? Drug Alcohol Depend 57:183-192.

Pinchevsky GM, Arria AM, Caldeira KM, Garnier-Dykstra LM, Vincent KB, and O'Grady KE. 2012. Marijuana exposure opportunity and initiation during college: parent and peer influences. Prev Sci 13(1):43-54. 10.1007/s11121-011-0243-4.

Rhodes T, Lilly R, Fernández C, Giorgino E, Kemmesis UE, Ossebaard HC, Naser L, Faasen I, and Spannow KE. 2003. Risk factors associated with drug use: the importance of 'risk environment'. Drugs: education, prevention and policy 10:303-329.

Ruggeri D. 2013. Marijuana price estimates and the price elasticity of demand. International Journal of Trends in Economics Management and Technology (I: TEMT) 2:31-36.

United Nations Office on Drugs and Crime. 2016. World Drug Report 2016. Available at http://www.unodc.org/wdr2016/ (accessed March 1, 2017).

United States. 2012a. Substance Abuse and Mental Health Services Administration Center for Behavioral Health Statistics and Quality, Bureau of the Census. 2010-2011 National Survey on Drug Use and Health National Maps of Prevalence Estimates, by State. Available at http://archive.samhsa.gov/data/NSDUH/2k11State/NSDUHsae2011/Maps/NSDUHsaeMa ps2011.htm\#TopOfPage (accessed November 12015 ).

United States. 2012b. Substance Abuse and Mental Health Services Administration. Center for Behavioral Health Statistics and Quality. Comparing and evaluating youth substance use estimates from the National Survey on Drug Use and Health and other surveys (HHS Publication No. SMA 12-4727, Methodology Series M-9). Rockville, MD: Substance Abuse and Mental Health Services Administration.

United States. 2012c. Office of National Drug Control Policy. Drug Availability Estimates in the United States. In: Executive Office of the President and Office of National Drug Control Policy, editor. Washington DC: Executive Office of the President.

United States. 2015. Department of Health and Human Services. Substance Abuse and Mental Health Services Administration. Center for Behavioral Health Statistics and Quality. National Survey on Drug Use and Health: 10-Year R-DAS (2002 to 2011). ICPSR34482v3. Ann Arbor, MI: Inter-university Consortium for Political and Social Research.

United States. 2016. Department of Health and Human Services. Substance Abuse and Mental Health Services Administration. Center for Behavioral Health Statistics and Quality. 2014 NATIONAL SURVEY ON DRUG USE AND HEALTH METHODOLOGICAL RESOURCE BOOK SECTION 13: STATISTICAL INFERENCE REPORT. Rockville, MD: Substance Abuse and Mental Health Services Administration.

United States. Library of Congress. 2003. Marijuana availability in the United States and its associated territories In: Federal Research Division Library of Congress, editor. Washington DC: Federal Research Division, Library of Congress.

Volkow N, and Li TK. 2005. The neuroscience of addiction. Nat Neurosci 8:1429-1430. 10.1038/nn1105-1429.

Vsevolozhskaya OA and Anthony JC. 2014. Confidence interval estimation in R-DAS. Drug Alcohol Depend 143:95-105. 10.1016/j.drugalcdep.2014.07.017.

Wagner F, and Anthony JC. 2002. Into the world of illegal drug use: exposure opportunity and other mechanisms linking the use of alcohol, tobacco, marijuana, and cocaine. Am J Epi 155(10):918-925. 
Figure 1

Reproduction of Figure 2 in the Library of Congress report on cannabis (marijuana) availability in the United States. (United States Library of Congress, 2003).

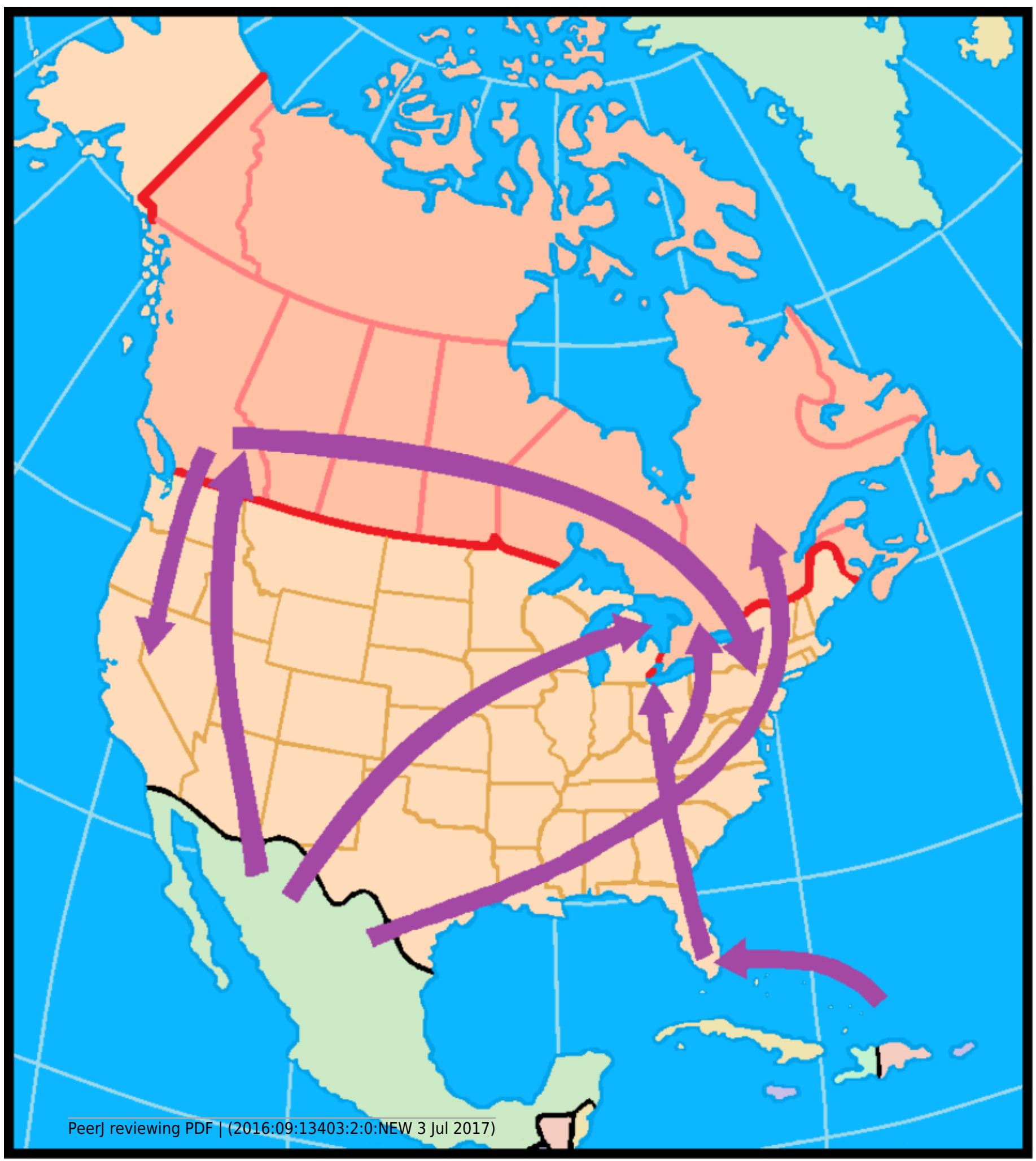




\section{Figure 2}

Region-specific annual incidence rate of cannabis use.

Data from United States National Surveys on Drug Use and Health 10-Year Restricted Data Analysis System, 2002-2011 (Unweighted $n \sim 420,000$ 12-24 year olds).

Note: Reported estimates and their $95 \%$ Cls (i.e. numbers in the right column) are rounded to the hundreds decimal point due to relatively small sample sizes in small regions.

Estimated Risk

(Per 100)

Region

$(95 \% \mathrm{Cl})$

North Atlantic Border

Pacific Border

Canada Border

Hawaii

Washington

Alaska

Mexico Border

Non-Border States

South Atlantic Border

Gulf of Mexico Border

Overall
$7(6,8)$

$7(5,8)$

$6(6,7)$

$6(5,7)$

$6(5,7)$

$6(5,7)$

$5(4,7)$

$5(5,6)$

$5(5,6)$

$5(4,6)$

$6(5,6)$

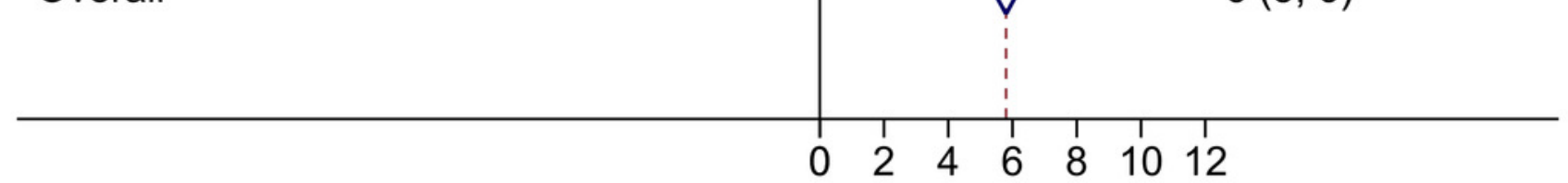

Annual Incidence of Cannabis Use (\%) 


\section{Figure 3}

Heat map representation of state-specific incidence rates.

Gradient heat map representation of each state of the United States in terms of its incidence rate for newly incident cannabis use as estimated for 12-to-24-year-old non-institutionalized civilian community residents. Data from the United States National Survey on Drug Use and Health, 2002-2011.

Note: This heat map helps to show that (1) neither the Mexico border states, nor the Gulf of Mexico states have exceptionally large cannabis incidence rates, (2) the northeastern region (including Vermont) has relatively large cannabis incidence rates, and (3) Utah has a relatively small cannabis incidence rate. Comparison of Figure 3 with Figure 4 may be useful.

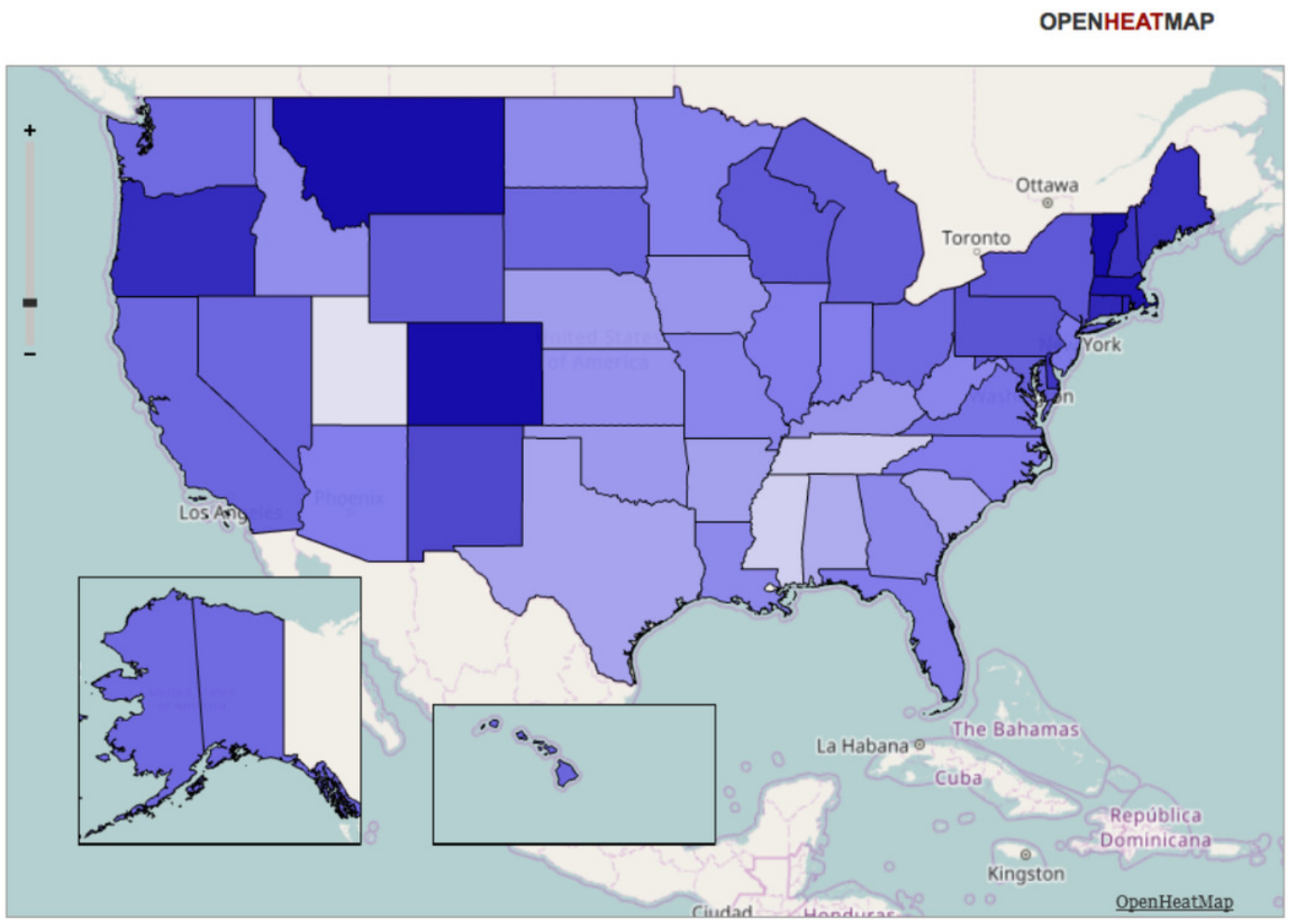

\begin{tabular}{l|l|l|l|} 
Incidence Rate & 3.00 & 5.50 & 8.00
\end{tabular} 


\section{Figure 4}

Cartogram representation of state-specific incidence rates.

Cartogram ('blob map') representation of each state of the United States in terms of relative size of its population incidence rate for newly incident cannabis use as estimated for 12-to-24-year-old noninstitutionalized civilian community residents. Data from the United States National Survey on Drug Use and Health, 2002-2011.

Note: This cartogram is oriented top (North), bottom (South), left (West), and right (East). It also helps to show that (1) neither the Mexico border states, nor the Gulf of Mexico states have exceptionally large cannabis incidence rates, (2) the northeastern region (including Vermont) has relatively larger cannabis incidence rates, and (3) Utah has a relatively small cannabis incidence rate. (Utah can be located in relation to the Southwest corner of the map where California has a Pacific coastline and shares a southern border with Mexico. California's southeast corner has a border with Arizona, above which is Nevada. Utah can be seen as an L-shaped state just above Arizona and to the East of Nevada.) Comparison of Figure 4 with Figures 1 and 3 may be useful.

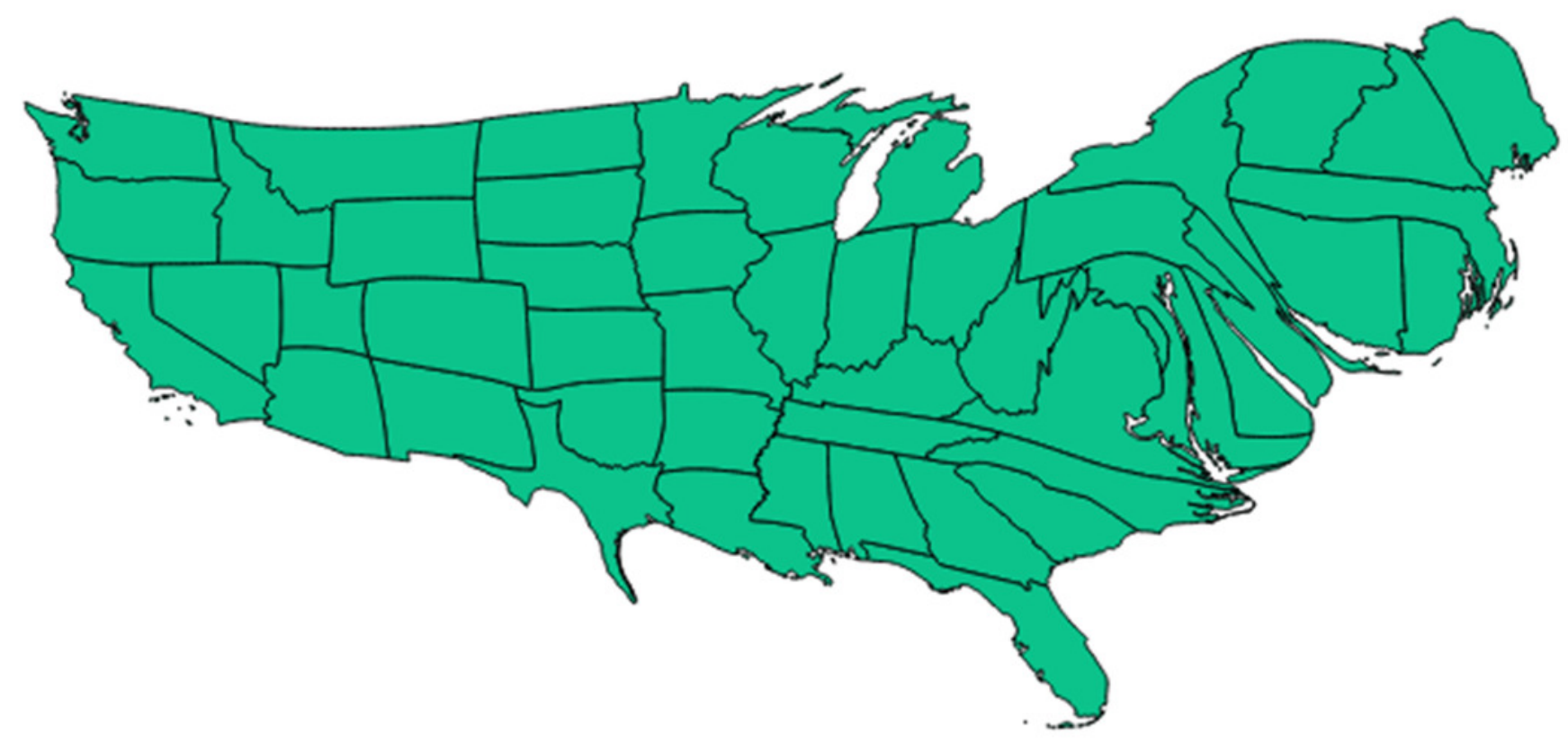

